\title{
The effect of Jupiter's mass growth on satellite capture
}

\section{Retrograde case}

\author{
E. Vieira Neto ${ }^{1, \star}$, O. C. Winter ${ }^{1}$, and T. Yokoyama ${ }^{2}$ \\ 1 Grupo de Dinâmica Orbital \& Planetologia, UNESP, CP 205 CEP 12.516-410 Guaratinguetá, SP, Brazil \\ e-mail: ernesto@feg.unesp.br \\ ${ }^{2}$ Universidade Estadual Paulista, IGCE, DEMAC, CP 178 CEP 13.500-970 Rio Claro, SP, Brazil \\ e-mail: tadashi@ms.rc.unesp.br
}

Received 13 June 2003 / Accepted 12 September 2003

\begin{abstract}
Gravitational capture can be used to explain the existence of the irregular satellites of giants planets. However, it is only the first step since the gravitational capture is temporary. Therefore, some kind of non-conservative effect is necessary to to turn the temporary capture into a permanent one. In the present work we study the effects of Jupiter mass growth for the permanent capture of retrograde satellites. An analysis of the zero velocity curves at the Lagrangian point $L_{1}$ indicates that mass accretion provides an increase of the confinement region (delimited by the zero velocity curve, where particles cannot escape from the planet) favoring permanent captures. Adopting the restricted three-body problem, Sun-Jupiter-Particle, we performed numerical simulations backward in time considering the decrease of $M_{4}$. We considered initial conditions of the particles to be retrograde, at pericenter, in the region $100 R_{4} \leq a \leq 400 R_{4}$ and $0 \leq e \leq 0.5$. The results give Jupiter's mass at the moment when the particle escapes from the planet. Such values are an indication of the necessary conditions that could provide capture. An analysis of these results shows that retrograde satellites would be captured as soon as they get inside the Hills' radius and after that they keep migrating toward the planet while it is growing. For the region where the orbits of the four "old" retrograde satellites of Jupiter (Ananke, Carme, Pasiphae and Sinope) are located we found that such satellites could have been permanently captured when Jupiter had between $62 \%$ and $93 \%$ of its present mass.
\end{abstract}

Key words. planets and satellites: general - astrometry - celestial mechanics

\section{Introduction}

All the giant planets have irregular satellites, with high eccentricity, high inclination (many are retrogrades) and are distant from the planet (more than 100 planetary radii). The vast majority of these satellites were discovered recently (Sheppard et al. 2001, 2002, 2003; Gladman et al. 2000, 2001; Holman et al. 2003). Due to their orbital characteristics such satellites are believed to have an origin different from the regular satellites, which would have been formed in a protosatellite disk around the parent planet. The most accepted idea is that they were formed elsewhere and were captured by the planet (Peale 1999).

The capture process can be divided into two phases. First, during an encounter with the planet, the candidate satellite is temporarily captured by the gravitational attraction of the planet. Then, some sort of non-conservative effect occurs to turn the capture into a permanent one. One of the main points of the first phase is the length of time a temporary capture lasts,

Send offprint requests to: O. C. Winter,

e-mail: ocwinter@feg.unesp.br

^ UNESP Post Doctor Program. since this time scale is relevant for the second stage to be accomplished.

In a previous paper (Vieira Neto \& Winter 2001) we have studied this problem of temporary gravitational capture for hypothetical satellites of Uranus. The adopted approach was to compute the capture times for a significant part of the initial conditions space. The results were presented in terms of graycoded diagrams of semi-major axis $(a)$ versus eccentricity $(e)$ taking fixed values for the other initial orbital elements. One of the important features presented in the results were the regions of quasi-periodic trajectories, trajectories that did not escape for an integration period of $10^{5}$ years.

Several mechanisms have been proposed to accomplish the second phase of the capture process. Among them are: the gas drag (Polack et al. 1979; Mckinnon \& Leith 1995; Saha \& Tremaine 1993); change of mass of the primaries (Heppenheimer 1975; Heppenheimer \& Porco 1977; Byl \& Ovenden 1975; Brunini 1995); orbital migration (Brunini 1995); collision between satellites (Colombo \& Franklin 1971; Horedt 1989; Goldreich et al. 1989); scattering by a regular massive satellite (Cline 1979; Tsui 1999, 2000).

In the present work we explore the possible contribution of a mechanism that occurred during the solar system formation, 

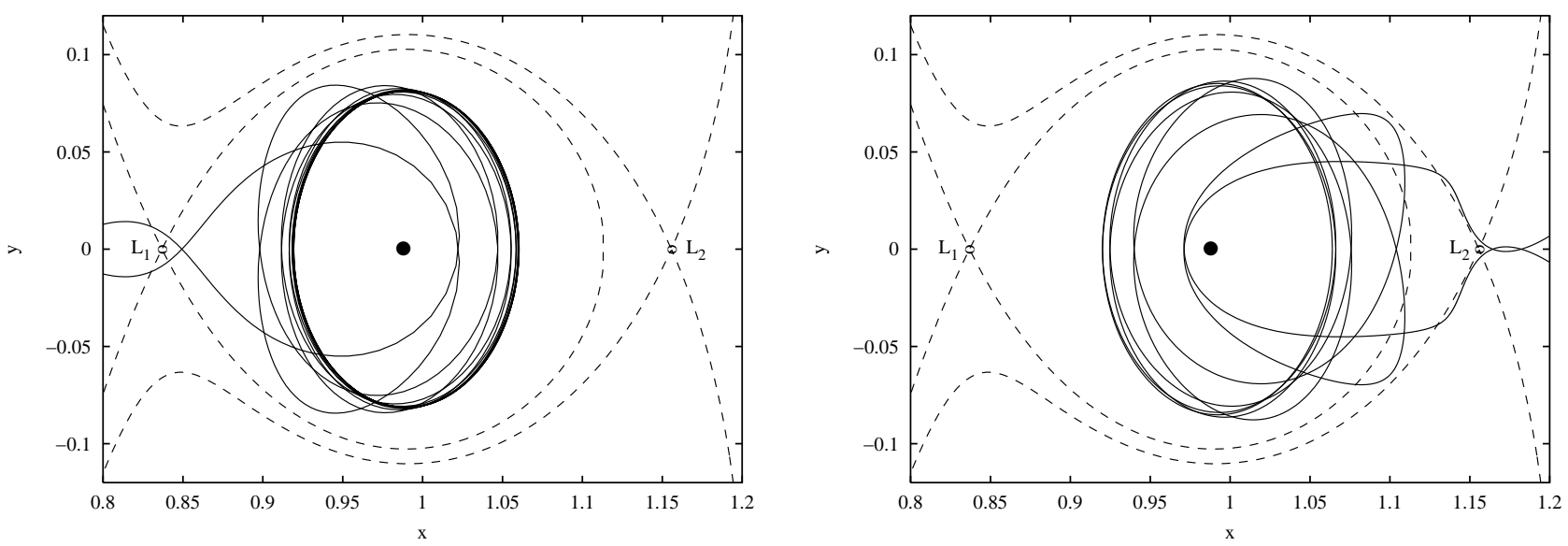

Fig. 1. Gravitational capture trajectories in the circular restricted three-body problem. The reference system is barycentric rotating with the primaries. On the left we see a trajectory that is temporarily captured and escapes from the $L_{1}$ side, on the right the trajectory is temporarily captured and escapes from the $L_{2}$ side. The dotted curves are the zero velocity curves at $L_{1}$ and at $L_{2}$.

the mass growth of Jupiter. The other possible mechanisms will be considered in future work. In the next section we discuss the change in the zero velocity curves at the Lagrangian point $L_{1}$ due to this mechanism, and its implications for the capture problem. Then, we perform an analysis of the effects of the planet's mass growth on its sphere of influence and on the semi-major axis of its satellites. The conclusion of this analysis leads to a condition for capture/escape of a satellite. In Sect. 3, we present our numerical simulations adopting the restricted three-body problem, Sun-Jupiter-Particle, taking into account the effects of Jupiters mass growth. The results show at which stage of Jupiters mass growth a given retrograde satellite would have been captured. The simulations for the case of the four retrograde satellites of Jupiter known of old are presented in Sect. 4. Our final comments are given in the last section.

\section{Effects due to mass change}

Considering the planar circular restricted three-body problem, the Jacobi constant can be given by (Szebehely 1967)

$C_{\mathrm{J}}=x^{2}+y^{2}+2\left(\frac{\mu_{1}}{r_{1}}+\frac{\mu_{2}}{r_{2}}\right)-\dot{x}^{2}-\dot{y}^{2}$

where $x, y, \dot{x}$ and $\dot{y}$ are the components of position and velocity in the barycentric rotating frame, $r_{1}$ and $r_{2}$ are the distances to the primaries, and $\mu_{1}$ and $\mu_{2}$ are the reduced masses. The zero velocity curves are the contour plots in the $x y$ plane for fixed values of $C_{\mathrm{J}}$ taking $\dot{x}=\dot{y}=0$.

A gravitational capture occurs when a particle initially orbiting the Sun gets close to the planet and stays temporarily orbiting it. The most relevant temporary gravitational captures are those of long duration. In such cases the particle enters the region defined by the zero velocity curve associated to the Lagrangian point $L_{1}$. The value of the Jacobi Constant, $C_{\mathrm{J}}$, of such a trajectory is close to, but less than the value at $L_{1}, C_{\mathrm{J}}\left(L_{1}\right)$, or even at $L_{2}, C_{\mathrm{J}}\left(L_{2}\right)$. So the particle is allowed to stay in the vicinity of the planet, but at some stage it will escape. In Fig. 1 we show two examples of capture trajectories; these trajectories had to pass the vicinities of the $L_{1}$ or $L_{2}$ to be captured.
The trajectory of Fig. 1a has a value of the Jacobi Constant between the values at $L_{2}$ and $L_{1}$, while the trajectory of Fig. 1b has a value of Jacobi Constant not much smaller than the value at $L_{2}$.

A temporary gravitational capture in the examples given in Fig. 1 could become permanent due to the mass change of one of the primaries (Heppenheimer \& Porco 1977). That would happen if the change in the mass produces a change in the zero velocity curve of the particle such that it is not allowed to escape anymore. In Fig. 2 we present the zero velocity curves associated to the Lagrangian point $L_{1}$ in the planetocentric $a \times e$ space, for a particle at pericenter. In this diagram such a curve divides the space into two regions. One (to the left of the curve), where particles are confined around the planet and will never escape. Another (to the right of the curve), where particles can stay temporarily around the planet, but will sooner or later escape.

Figure 2 shows the locations of the zero velocity curves associated to the Lagrangian point $L_{1}$ for the case of Jupiter with its present mass $\left(320 M_{\oplus}\right)$ and with $10 \%$ of its present mass $\left(32 M_{\oplus}\right)$. The area of the region defined by the zero velocity curve increases as the mass grows. So, some trajectories that had $C_{\mathrm{J}}<C_{\mathrm{J}}\left(L_{1}\right)$ while Jupiter was growing could have $C_{\mathrm{J}}>C_{\mathrm{J}}\left(L_{1}\right)$ when Jupiter was completely formed. Therefore, based only on this simple analysis the mass accretion mechanism is favorable to the capture process. It is important to note that in the case of retrograde orbits there are stable trajectories even outside the zero velocity curve associated to $L_{1}$. They are quasi-periodic orbits associated to a family of periodic orbits (Vieira Neto \& Winter 2001).

The mass variation of Jupiter produces at least two relevant effects. One of them is the change in its gravitational sphere of influence. For instance, as the planet mass increases its Hill's radius,

$R_{\text {Hill }}=\left(\frac{\mu_{2}}{3}\right)^{\frac{1}{3}}$,

also increases. The other correlated effect produced by the mass variation of the planet is the change on the orbital semi-major 


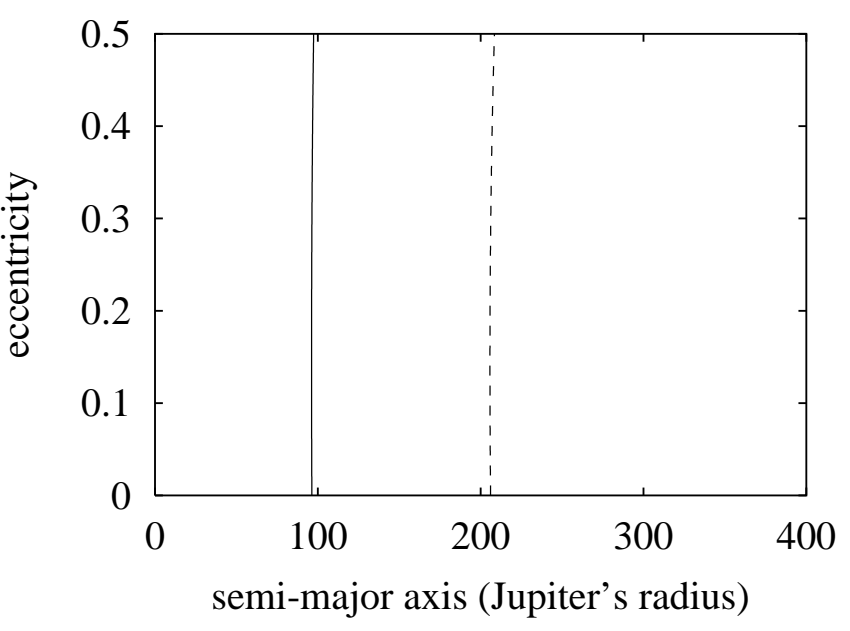

Fig. 2. Zero velocity curves associated to the Lagrangian point $L_{1}$ in the planetocentric $a \times e$ space at pericenter in retrograde orbit. The line to the right corresponds to the case of Jupiter with its present mass $\left(320 M_{\oplus}\right.$ ) and the left line corresponds to the case of Jupiter with $10 \%$ of its present mass $\left(32 M_{\oplus}\right)$.

axis $(a)$ of its satellites. This change follows the relation given by Jeans (1961)

$a \mu_{2}=$ constant

The evolution of the present dynamical system is reversible in time, i.e. when the equations of motion are integrated backward in time for a length of time $\Delta t$ and then forward in time for this same amount of time the system returns to the same state of the initial conditions. So, we preferred to make the analysis going backwards in time. Therefore, we considered a particle that begins as a bounded satellite of Jupiter, with its present mass, then we follow the dynamical evolution of this particle to the past. As the mass of the Jupiter decreases the semi-major axis of the satellite increases. As a consequence the gravitational influence of the planet on the satellite decreases and when the satellite reaches a radial distance from the planet such that its gravitational influence is not enough to keep it bound, it will escape (Fig. 3).

According to this analysis, at a given stage of the mass evolution of the planet there is a critical radial distance beyond which the satellite escapes. In Fig. 4 we present a diagram of orbital radius versus $M_{4}$. The grey area corresponds to the condition where the object would be orbiting the planet as a satellite, delimited by what we called the Gravitational Capture Boundary. There is also a line labeled Jean's Relation which gives an example of the evolution of the orbital radius as a function of $M_{4}$. As the mass of the planet changes the particle's orbital radius goes from inside the grey area to outside, or the other way around, depending on the arrow of the time. Therefore, once the Gravitational Capture Boundary is known, one can identify at which stage of the planet's mass growth a given satellite was captured.

\section{Numerical simulations}

The effect of planetary growth on the dynamics of planetesimals has recently been considered in the literature.

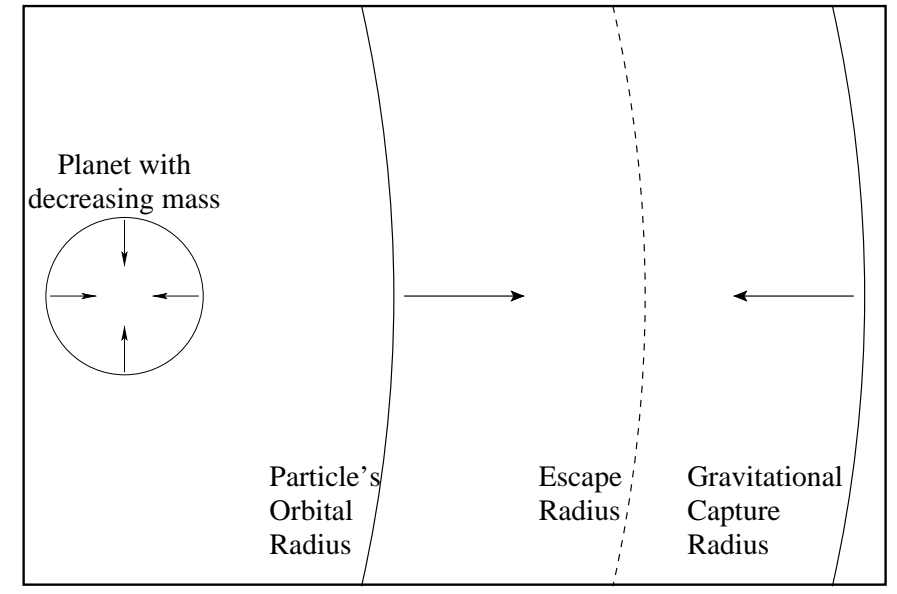

Fig. 3. Diagram illustrating the process involved in a planet-satellite system when the planet's mass is decreasing. The particle's orbital radius increases while the gravitational capture radius of the planet decreases. The distance at which these two radii coincide is the escape radius.

Marzari \& Scholl (1998) showed numerically that an increase in $M_{4}$ could cause the capture of planetesimals into librating Trojan orbits. Fleming \& Hamilton (2000) explored analytically and numerically the dynamical effects of Jupiter's accretion on its Trojan companions.

In order to verify the actual dynamical effects of Jupiters mass growth on the capture problem we performed some numerical simulations. We adopted the planar, circular, restricted three-body problem, Sun-Jupiter-Particle, with a variation of the mass of Jupiter. Since the evolution of this dynamical system is reversible in time we conducted simulations taking initial conditions of particles inside a region delimited by $100 R_{4} \leq a \leq 400 R_{4}$ and integrated backwards in time. The particle is initially bounded as a satellite of Jupiter, with its present mass, then we follow the dynamical evolution of this particle to the past as the mass of Jupiter decreases. The simulations were interrupted in two situations: i) the 2-body energy, Jupiter-particle, changed from negative to a positive value (escape/capture); ii) the particle collided with Jupiter (collision). From such simulations we determined the time at which the escape occurred for each particle's initial condition. Assuming that the present dynamical system adopted fairly represents the history of this satellite system, the results tell us at which stage of Jupiters mass growth an irregular retrograde satellite would have been captured by Jupiter.

We adopted the variation of $M_{4}$ to be linear with time on time scales ranging from $10^{3}$ to $10^{5}$ years (the results are similar if an exponential growth is adopted (Fleming \& Hamilton 2000; Yokoyama et al. 2003)). The initial conditions were such that the particles were considered at pericenter in opposition within the ranges $0 \leq e \leq 0.5$ and $100 R_{4} \leq a \leq 400 R_{4}$, where $R_{4}$ is Jupiter's equatorial radius. Most of the numerical simulations were made considering planar retrograde orbits, $I=180^{\circ}$.

The results show the mass of Jupiter at the moment when the particle escaped from the planet (Fig. 5). The time scale for $M_{4}$ to decrease from its present value to $10 \%$ of it was 


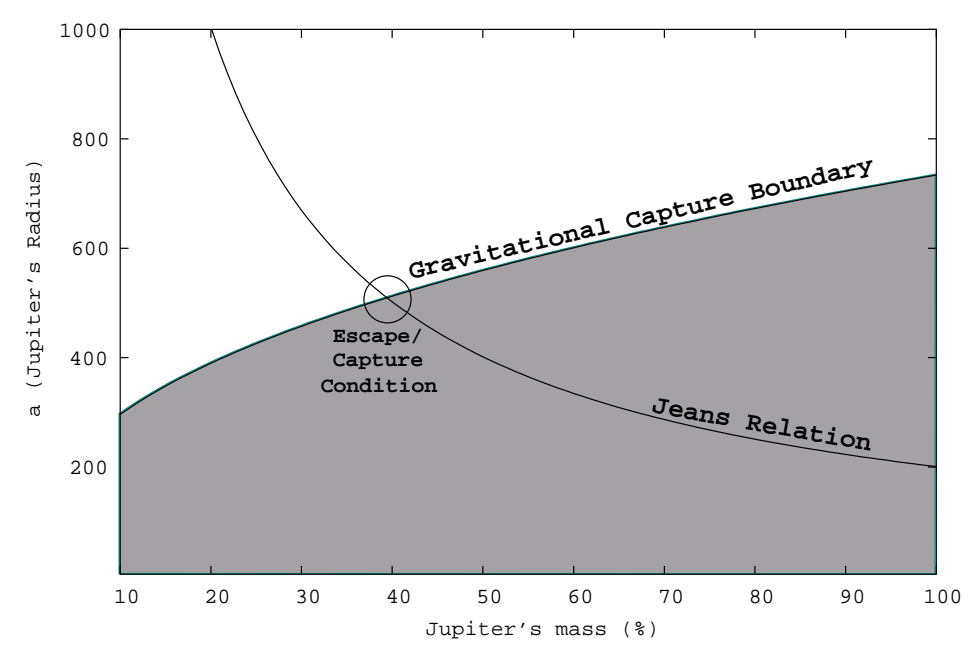

Fig. 4. Diagram of the orbital radius versus $M_{4}$. The grey area corresponds to the condition where the object would be orbiting the planet as a satellite. The line labeled Jean's Relation gives the evolution of the orbital radius as a function of the mass of Jupiter for an object that would be orbiting as a satellite of Jupiter at the present moment in a circular orbit with $a=100 R_{4}$. The particle's orbital radius increases while the gravitational capture radius of the planet decreases. The distance at which these two radii coincides is the escape radius.

$10^{3}$ years in Fig. $5 \mathrm{a}, 10^{4}$ years in Fig. $5 \mathrm{~b}$ and $10^{5}$ years in Fig. 5c. The structure of the figures show that the time scale for mass growth is not a very relevant factor for the escape/capture process. What actually dominates the process is the value of the mass of the planet at the moment of escape. Such values give an indication of the necessary conditions that could provide the permanent capture. Nevertheless, we notice that as the time scale gets larger the escape occurs earlier. In other words, there is a slight dependence of the mass growth time scale on the stage of the mass growth at which the particle escaped from the planet. The difference of $M_{4}$ values between the results for time scales $10^{3}$ years and $10^{4}$ years is of the order of $5 \%$ and between $10^{4}$ years and $10^{5}$ years is of the order of $3 \%$. That is an indication that for longer time scales $\left(10^{7}, 10^{8}\right.$ years $)$ the results are not much different from those presented here.

The general structures presented in Figs. 5a-c show that the farthest from the planet the particle is the sooner it escapes, as expected. The shifted band that appears in Figs. 5 for low values of eccentricity $(e \leq 0.1)$ is associated to a family of periodic orbits (Winter \& Vieira Neto 2001). This family of periodic orbits retards the escape of the satellites that are librating around it and also affect the trajectories nearby. The effect of this family of periodic orbits decreases as the time scale for mass variation increases (the band narrows). The general structure shown in Figs. 5a-c means that there is an explicit relation between the planets' mass and the initial semi-major axis and eccentricity of the particle. Using an implementation of the nonlinear least-squares through a Marquardt-Levenberg algorithm we determined the relation

$$
\begin{aligned}
M(a, e)= & {[0.0387665 \pm 0.0021] } \\
& +\left[0.001444 \pm 1.3 \times 10^{-5}\right] a \\
& +[0.1737 \pm 0.0089] e \\
& +\left[0.002429 \pm 1.6 \times 10^{-5}\right] a e \\
& -\left[7.81 \times 10^{-7} \pm 2.4 \times 10^{-8}\right] a^{2} \\
& -[0.3959 \pm 0.0131] e^{2},
\end{aligned}
$$

where $a$ is given in Jupiter's radius and $M$ in $M_{4}$. Equation (4) was generated from the data of the results found in the case of time scale $10^{5}$ years, with $e \geq 0.1$. This equation reproduces very well the results found through numerical integrations as can be verified in the contour plots shown in Fig. 5e.

To investigate the effects due to the orbital inclination we also simulated the dynamical system for initial conditions of retrograde orbits out of the plane. All the known retrograde satellites have inclinations between $140^{\circ}$ and $180^{\circ}$. Yokoyama et al. (2003) and Carruba et al. (2002) showed that satellites with inclinations between $55^{\circ}$ and $130^{\circ}$ would be highly unstable. Due to the Kozai resonance they would reach eccentricity high enough to enter the region of the galilean satellites and be removed by them.

Therefore, we performed numerical simulations considering the cases of $I=140^{\circ}$ and $I=160^{\circ}$, with a mass growth time scale of $10^{4}$ years. The results are presented in Figs. 5d and $f$, respectively. We can see the changes due to the inclination by looking at the left column of Fig. 5. From top to bottom, we note that the distortion produced by the family of periodic orbits $\left(I=180^{\circ}\right)$ disappears as the inclination decreases. On the other hand, the structure of the figures is almost the same for $e \geq 0.3$.

In order to better understand the structure shown in Fig. 5, we analyzed in detail a representative set of initial conditions of planar retrograde orbits for each of the three time scales given in Figs. 5a-c. We considered the set composed by nine initial conditions with $I=180^{\circ}$ and given by the combinations of $a=100 R_{4}, 225 R_{4}, 350 R_{4}$ and $e=0.10,0.25,0.40$. The semi-major axis of the orbits evolve as predicted by the Jeans relation (Eq. (3)), as can be seen in the examples presented in Fig. 6. As the mass of Jupiter decreases the eccentricity of the orbits increases the amplitude of oscillation. Notice that the rate of increase in the amplitude of oscillation of the eccentricity is proportional to the initial eccentricity (Fig. 6).

The combination of the evolution of the semi-major axis and the eccentricity produces an increase of the orbital radius 

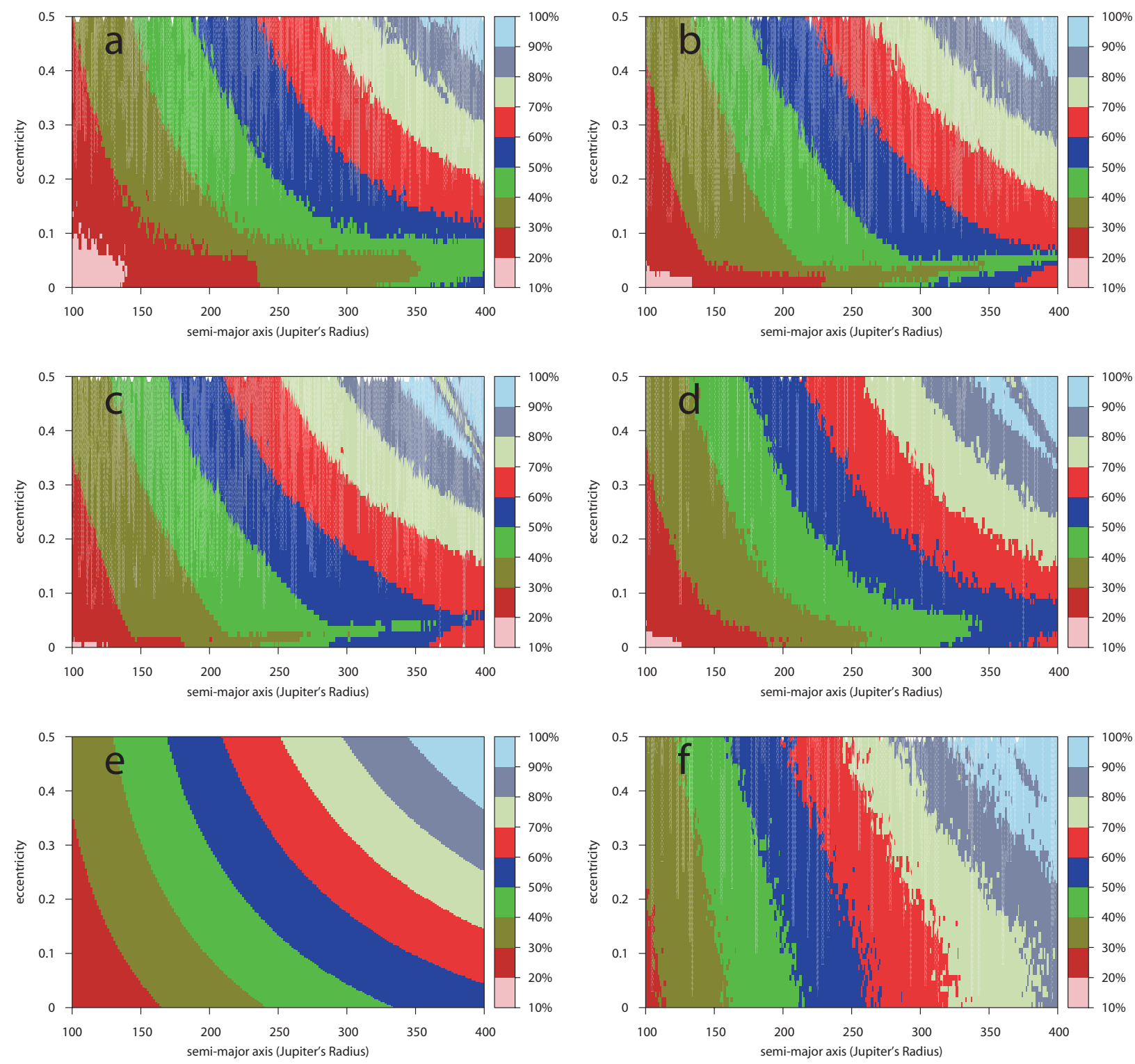

Fig. 5. Mass of Jupiter at the moment of escape for particles with initial conditions in the planetocentric $a \times e$ space. Initially Jupiter has its present mass $\left(320 M_{\oplus}\right)$ and the orbits were integrated backward in time with a decreasing mass of Jupiter. In the first three figures the orbital inclination considered was $180^{\circ}$ and the time scale for the mass to decrease from its present mass to $10 \%$ of its mass was: a) $10^{3} \mathrm{yr}$, b) $10^{4} \mathrm{yr}$ and c) $10^{5} \mathrm{yr}$. There are also two figures where were adopted the time scale of $10^{4} \mathrm{yr}$, but with orbital inclinations: d) $160^{\circ}$ and f) $140^{\circ}$. The only figure that is not a result from numerical integration is Fig. e). It presents contour plots given by Eq. (4).

of the particle and of its amplitude of oscillation. In general, the orbital radii of the particles evolve as shown in the sample given in Fig. 7.

Following the idea that there is a critical distance to the planet, defined as the Gravitational Capture Boundary (Fig. 4), beyond which the particle will escape, we determined this boundary from the results of our simulations. In the case of Jupiters mass growth time scale of $10^{5}$ years, this boundary is very well fitted by one Hill's radius (Eq. (2)), as can be seen in the plots of Fig. 7. In the cases of time scales $10^{4}$ and $10^{3}$ years the best fits are $1.1 R_{\mathrm{Hill}}$ and $1.4 R_{\mathrm{Hill}}$, respectively. Therefore, the results suggest that the Gravitational Capture Boundary decreases as the mass growth time scale increases, but it also suggests a convergence to a value close to one Hill's radius. This is not a naturally expected result since the existence of stable retrograde orbits much further than this limit is well known (see for instance Vieira Neto \& Winter 2001 and references therein).

\section{4. "Old" retrograde satellites}

In this section we considered separately the case of the four retrograde satellites of Jupiter that have been known for more than half a century (Ananke, Carme, Pasiphae and Sinope). Sheppard \& Jewitt (2003b) classified all the irregular retrograde satellites of Jupiter into three groups. One group has Ananke as the parent body, other is associated to Carme and a third group is associated to Pasiphae and Sinope. Therefore, 

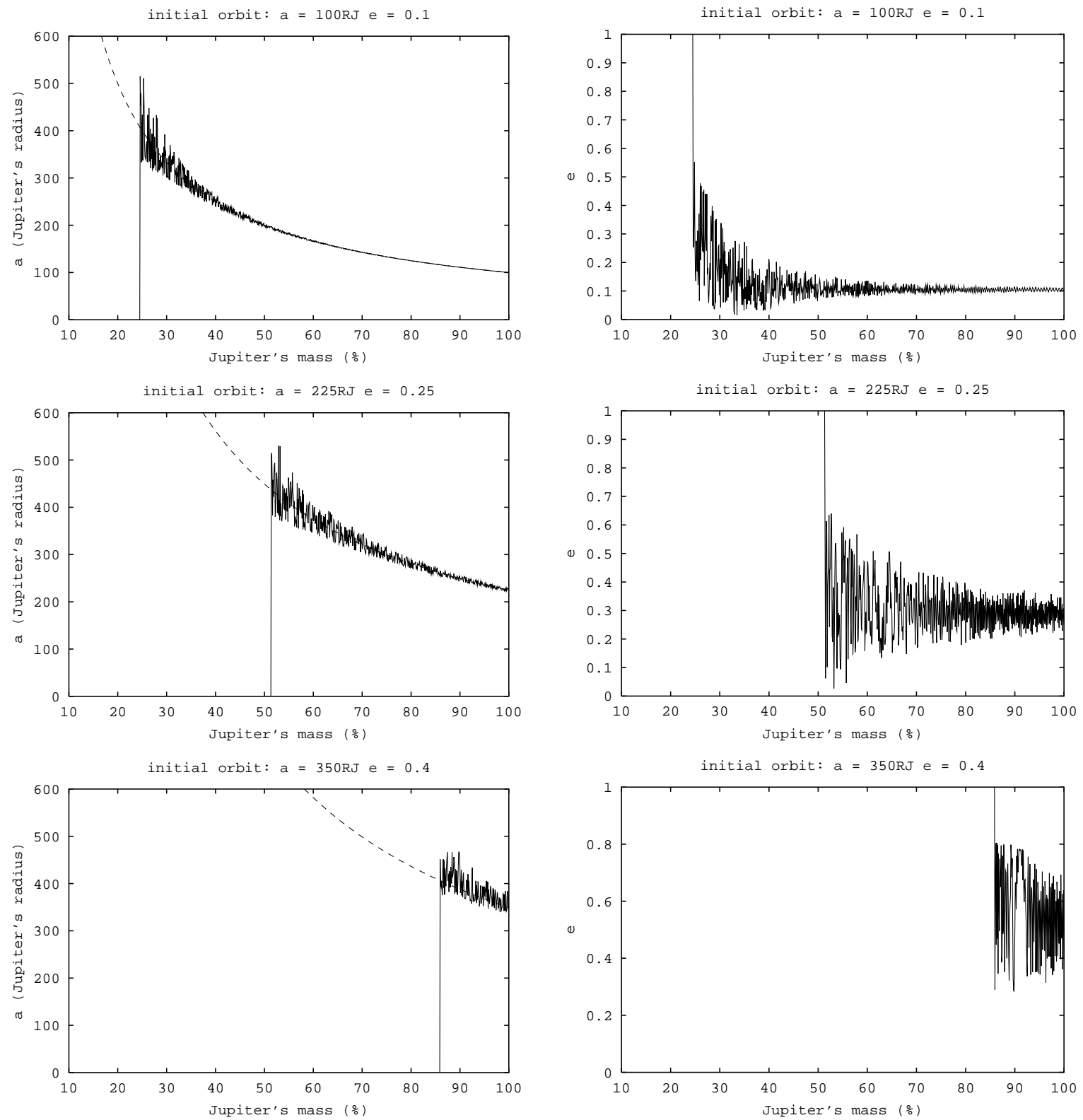

Fig. 6. Evolution of the semi-major axis (left column) and eccentricity (right column) of a sample of particles as a function of Jupiter's mass. This is a representative sample from the simulations with mass variation time scale equals to $10^{5}$ years and $180^{\circ}$ of inclination. The initial conditions are: $a=100 R_{4}$ and $e=0.10$ (top); $a=225 R_{4}$ and $e=0.25$ (middle); $a=350 R_{4}$ and $e=0.40$ (bottom). The dotted lines are given by Jeans' relation, Eq. (3).

the results presented in this section can be used as an estimate for all the three groups.

Considering the tridimensional problem, we numerically simulated the trajectories for sets of initial conditions around their present orbits. As the orbits of these satellites are known to vary significantly (Saha \& Tremaine 1993) we decided to take their osculating orbital elements at a given epoch, $a_{0}, e_{0}$ and $I_{0}$ and use a set of 27 initial conditions for each satellite given by the combinations of $a_{0} \pm 0.01 a_{0}, e_{0} \pm 0.1 e_{0}$ and $I_{0} \pm 0.01 I_{0}$. We considered two different time scales for Jupiters mass growth: $10^{5}$ years and $10^{7}$ years.

The results indicate that these satellites could have been captured permanently during Jupiter's mass growth. Table 1 gives the stage at which Jupiter's evolution should be for
Table 1. Orbital elements of some retrograde satellites of Jupiter and the stage during Jupiters mass growth at which the satellite could have been captured (last two columns).

\begin{tabular}{cccccc}
\hline \hline Satellite & $a_{0}$ & $e_{0}$ & $I_{0}$ & \multicolumn{2}{c}{ Jupiter's Mass } \\
& $\left(R_{4}\right)$ & & & $\left(10^{5} \mathrm{yr}\right)$ & $\left(10^{7} \mathrm{yr}\right)$ \\
\hline Carme & 316.5 & 0.21 & $163^{\circ}$ & $62-70 \%$ & $65-74 \%$ \\
Ananke & 296.9 & 0.17 & $147^{\circ}$ & $63-72 \%$ & $68-74 \%$ \\
Sinope & 331.9 & 0.28 & $153^{\circ}$ & $68-82 \%$ & $72-85 \%$ \\
Pasiphae & 329.1 & 0.38 & $147^{\circ}$ & $75-92 \%$ & $77-93 \%$ \\
\hline
\end{tabular}

capture to occur. The results are in good agreement with those presented in Figs. 5d and f, for $I=160^{\circ}$ and $I=140^{\circ}$, 

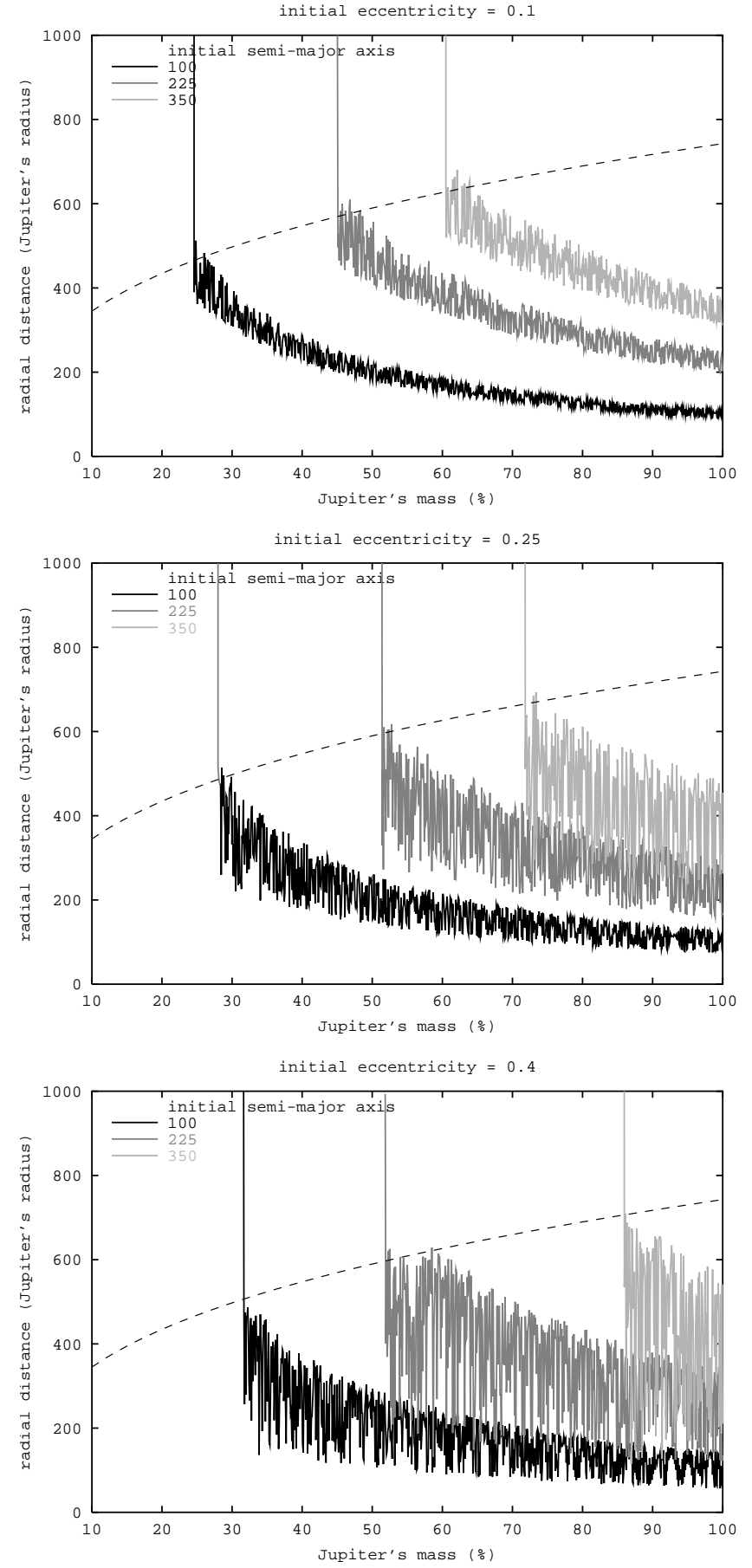

Fig. 7. Evolution of the orbital radius of a sample of particles as a function of the mass of Jupiter. This is a representative sample from the simulations with mass variation time scale equals to $10^{5}$ years. The initial conditions are $a=100 R_{4}$ (black), $a=225 R_{4}$ (gray) and $a=350 R_{4}$ (light gray) with $e=0.10$ (top), $e=0.25$ (middle) and $e=0.40$ (bottom). The dotted lines correspond to the Hill's radius as a function of the planet's mass.

respectively Pasiphae would have been the last to be captured. This is due to the fact that at present it has the greatest apocentric distance. The range of $M_{4}$ values for time scale $10^{5}$ years is not much different from that for $10^{7}$ years, at most $5 \%$ of Jupiter' s mass.
We also investigated the possible contributions to this escape/capture process due to the orbital eccentricity and inclination of Jupiter and the gravitational influence of Saturn and Uranus. In order to make this investigation we performed numerical integrations of the restricted n-body system, SunJupiter-Saturn-Uranus-Particle, going backward in time and taking into account the decreasing of Jupiter's mass for time scales of $10^{5}$ and $10^{7}$ years. The results found for these four satellites indicate that there is no significant contribution from these such perturbations.

\section{Conclusions}

We have presented an analysis of the gravitational escape/capture process of retrograde satellites taking into account the effect of Jupiter's mass growth. This analysis shows the existence of a Gravitational Capture Boundary beyond which a satellite would escape. Our numerical simulations agree very well with this analysis. The Gravitational Capture Boundary was determined from the numerical results. It is found that this Boundary depends on the time scale of the mass growth. However, as the time scale increases the Boundary seems to converge to one Hill's radius.

In the numerical simulation we confirmed that Jupiter's mass growth is an efficient mechanism for the capture of retrograde satellites. Our results show at which stage of Jupiter's mass growth a given retrograde satellite could have been captured. The actual values, given in these results, show a weak dependence on the time scale of the mass growth. In the case of the known retrograde satellites of Jupiter we found that such satellites could have been permanently captured when Jupiter had between $62 \%$ and $93 \%$ of its present mass. These values can also be used as an estimate for the groups of newly discovered retrograde satellites of Jupiter (Sheppard \& Jewitt 2003b).

The effects of Jupiter's eccentricity and inclination, and also of the planetary perturbation of Saturn and Uranus are not significant. We did not simulate the dynamical system including the Galilean satellites. It seems that they do not contribute much to the dynamics of distant satellites. They might actually contribute only during the very final stage, just before the escape of the particles. They would affect the particles when they reach very high eccentricity before escape (see the sample of the evolution of $e$ in Fig. 6).

In the present work we explored the contribution of the planet's mass growth effect alone. Nevertheless, other effects have to be analyzed and combined with the present one to indicate the most probable scenario that would have generated irregular satellites.

Acknowledgements. We would like to thank an anonymous referee for helpful comments and suggestions that significantly improved the paper. This work was funded by Fapesp (proc. 02/00344-7) and CNPq. These supports are gratefully acknowledged.

\section{References}

Brunini, A. 1995, Earth Moon and Planets, 71, 281

Byl, J., \& Ovenden, M. W. 1975, MNRAS, 173, 579 
Carruba, V., Burns, J. A., Nicholson, P. D., et al. 2002, Icarus, 158, 434

Cline, J. K. 1979, Cel. Mec. Dyn. Astron., 19, 405

Colombo, G., \& Franklin, F. A. 1971, Icarus, 15, 186

Fernandez, J. A., \& Ip, W.-H. 1984, Icarus, 58, 109

Fernandez, J. A., \& Ip, W.-H. 1995, Planet. Space Sci., 44, 431

Fleming, H. J., \& Hamilton, D. P. 2000, Icarus, 148, 479

Gladman, B., Kavelaars, J. J., Holman, M., et al. 2000, Icarus, 147, 320

Gladman, B., Kavelaars, J. J., Holman, M., et al. 2001, Nature, 412, 163

Goldreich, P., Murray, N., Longaretti, P. Y., \& Banfield, D. 1989, Science, 245, 500

Gomes, R. 1997, AJ, 114, 396

Heppenheimer, T. A. 1975, Icarus, 24, 172

Heppenheimer, T. A., \& Porco, C. 1977, Icarus, 30, 385

Holman, M., Kavelaars, J., Grav, T., et al. 2003, IAU Circ., 8047

Horedt, G. P. 1989, A\&A, 209, 411

Jeans, J. H. 1961, Astronomy and Cosmogony (New York: Dover)

Malhotra, R. 1993, Nature, 365, 819
Malhotra, R. 1995, AJ, 110, 420

Marzari, F., \& Scholl, H. 1998, Icarus, 131, 41

Mckinnon, W. B., \& Leith, A. C. 1995, Icarus, 118, 392

Peale, S. J. 1999, ARA\&A, 37, 533

Polack, J. B., Burns, J. A., \& Tauber, M. E. 1979, Icarus, 37, 587

Saha, P., \& Tremaine, S. 1993, Icarus, 106, 549

Sheppard, S. S., Jewitt, D. C., Fernandez, Y., Magnier, E., \& Marsden, B. 2001, IAU Circ., 7555

Sheppard, S. S., Jewitt, D. C., Kleyna, J., Marsden, B., \& Jacobson, R. 2002, IAU Circ., 7900

Sheppard, S. S., Jewitt, D. C., Marsden, B., et al. 2003, IAU Circ., 8116

Sheppard, S. S., \& Jewitt, D. C. 2003, Nature, 423, 261

Szebehely, V. 1967, Theory of Orbits (New York: Academic Press)

Tsui, K. H. 1999, Planet. Space Sci., 47, 917

Tsui, K. H. 2000, Icarus, 148, 139

Vieira Neto, E., \& Winter, O. C. 2001, AJ, 122, 440

Winter, O. C., \& Vieira Neto, E. 2001, A\&A, 377, 1119

Yokoyama, T., Santos, M. T., Cardin, G., \& Winter, O. C. 2003, A\&A, 401,763 